\title{
Genome-wide association studies: powerful tools for improving drug safety and efficacy
}

'The power offered by GWAS, if applied for combining datasets regarding drug response from several thousands of individuals, and when focused on drugs with the most severe or common adverse events, may allow the development of diagnostics offering better prediction of drug response.'

We live in an exciting era for genetics! This is a direct consequence of the availability of powerful and affordable genotyping technologies, along with the development of robust computational methods for analyzing large genetic datasets from patient cohorts. Technology has improved to the point where assessment of $1,000,000$ SNPs across the human genome is a 'normal' task. The present issue of Pharmacogenomics includes a focus on genome-wide association studies (GWAS). The last 3 years have seen an unprecedented number of publications reporting on new GWAS, most of them concerning the discovery of new disease risk alleles [1-3]. Hundreds of recently published GWAS have opened new insights on genetic risk factors for a wide variety of complex human diseases, from diabetes and asthma to multiple sclerosis and several types of cancer. It remains to be seen to what extent this wealth of novel information on disease risk alleles will go toward improving healthcare.

\section{"Relatively few GWAS on drug response were so far published compared with the large numbers of disease risk GWAS. However, from those published, it is evident that the potential for gaining insight into the pharmacogenomics of ADRs...is very large."}

Most disease risk alleles so far discovered by GWAS have relatively small effects on disease susceptibility and their clinical value remains unclear. Often the clinical significance or relevant therapeutic targets of the discovered alleles are not known, so that knowledge regarding carrying these alleles may in most cases be the start of a hypothesis testing pathway. Yet, in the long run there might emerge some clinical use for risk allele data, when the information might allow personalized choice of therapeutics according to knowledge regarding the defective pathways most closely implicated in disease pathology for the individual patient. Such clinical applications may still be many years away, as even in cases where new therapeutics will be developed based on newly discovered risk alleles and their corresponding protein targets, it typically takes at least one decade for a new drug to reach the market.

The question then arises, what types of GWAS will be more likely to lead to clinical applications in the foreseeable future? GWAS are still costly, due to the need for large (typically over 1000) patient cohorts and to the fact that recruitment costs are not decreasing (even though genotyping costs continue to fall). A relatively less explored area in GWAS, and one where we feel that clinical applications may arrive much sooner compared with information regarding carrying disease risk alleles, is the study of pharmacogenetics - the genetic contribution to drug response. More specifically, GWAS could be harnessed for improving drug response - both safety and efficacy - for many current medicines. Drugs are often not safe enough: according to a 2006 report by the Centers for Disease Control (CDC) adverse drug reactions (ADRs) account for nearly $7 \%$ of all hospital admissions in the USA, with even higher frequencies seen in small children and the elderly [4,5]. Similarly, high ADR rates were observed in the UK [6]. While much knowledge has accumulated over the last two decades regarding genetic factors implicated in ADRs, mostly affected by polymorphic alleles of genes coding for drug-metabolizing enzymes (DME) and drug transporters, applying this knowledge in the clinic suffers from several barriers, which include a lack of reliable data on the genetics of drug response $[7,8]$. GWAS, thanks to their statistical power and lack of presumptions, offer the potential for bridging this knowledge gap through their capacity to scan the entire human genome in very large patient cohorts [9].

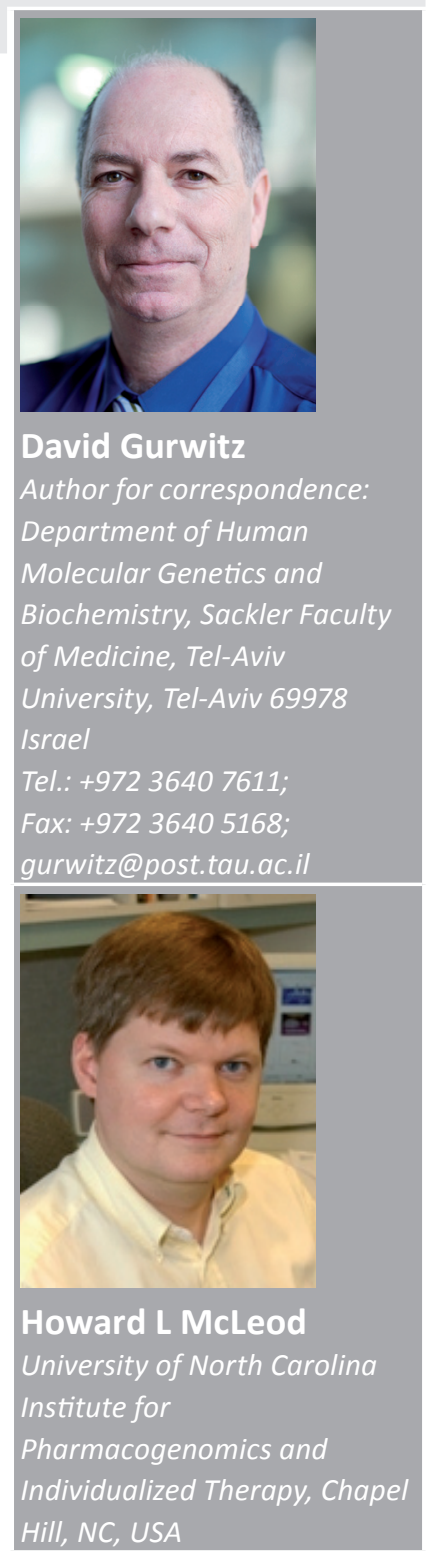

future medicine $^{\text {pind }} \mathbf{f S g}$ 
Relatively few GWAS on drug response were so far published compared with the large numbers of disease risk GWAS. However, from those published, it is evident that the potential for gaining insight into the pharmacogenomics of ADRs, and hence for developing diagnostics to aid in drug and dosage choices, is very large. A notable GWAS example is the recent observation that polymorphism of CYP2C8, coding for a well-known cytochrome P450 enzyme, is a predictor of bisphosphonate-related osteonecrosis of the jaw in multiple myeloma [10]. The finding that a DME was implicated in this ADR was a surprise, as bisphosphonates are not metabolized and are excreted intact. This suggests that CYP2C8 is involved in biological pathways of relevance to this ADR. Obviously, this finding would not have been possible without the power - and the lack of presumptions - offered by GWAS. Another fine example is the recent GWAS observation that genes associated with the $\gamma$-aminobutyric acid (GABA) receptor signaling pathway are involved in the common neuroleptic-induced ADR of tardive dyskinesia [11].

\section{"The challenge is finding an in vitro response phenotype that would reliably reflect in vivo drug efficacy."}

Moreover, drugs are often not effective enough for easing the symptoms they are meant to treat. Lack of efficacy of many current drugs is another major public health concern, considering the escalating costs of medicines and the time it may take to adjust a medicine for a patient who is meanwhile hospitalized or away from work. Lack of efficacy, in particular, is a burning issue for neurological and psychiatric diseases, where the lack of biomarkers to assist the physician in drug choice often means that therapy continues to be a matter of 'trial and error' $[12,13]$. Sadly, it seems that too little research is done for improving our knowledge regarding genetic determinants of drug efficacy; this is true in particular for generic drugs where intellectual property rights (IPR) aspects mean that there are little incentives for the private sector to develop the needed knowledge-base and diagnostics.

Things are not as bleak for costly drugs still enjoying patent protection, for which funding by pharma have allowed some novel drug efficacy GWAS, for example, concerning the efficacy of recombinant $\beta$-interferon, enjoying global sales of approximately US $\$ 5$ billion, in treating multiple sclerosis. $\beta$-interferon therapy is widely used for multiple sclerosis patients, but only about half of them benefit from this therapy. Indeed, these recent studies yielded some candidate genes, offering hopes that some diagnostics for personalizing drug choice for multiple sclerosis would follow [14]. Further recent GWAS on drug efficacy include reports on the efficacy of anti-TNF treatment in rheumatoid arthritis [15], smoking cessation with bupropion hydrochloride or nicotine replacement therapy [16] and methylphenidate in treating children with attention-deficit-hyperactivity disorder [17].

A novel advantage for GWAS of drug response (as opposed to GWAS for drug safely) is the potential for using cultured cell lines for gaining preliminary insights on genes associated with treatment efficacy, thereby saving (or rather, postponing for later research stages) the huge costs of recruiting large patient cohorts. The challenge is finding an in vitro response phenotype that would reliably reflect in vivo drug efficacy. This is relatively straight-forward when searching for genes related to the efficacy of anticancer drugs: those implicated in a more robust in vitro antiproliferative or pro-apoptotic response in cancer cell lines are more likely to reflect genetic markers for favorable therapeutic response in cancer patients (obviously this in itself is not informative regarding the drug safety in patients with the 'favorable' genetics for treatment efficacy). Examples for such preliminary studies - so far performed only in cell lines and hopefully to be confirmed soon in clinical trials - include GWAS on genes implicated in cytotoxicity of the commonly used anthracycline chemotherapeutic daunorubicin [18]; genes related to tamoxifen or aromatase inhibitors resistance in breast cancer cell lines [19]; and genes explaining response to lapatinib, an inhibitor of EGF receptor (EGFR) and HER2 tyrosine kinases [20]. One may hope that in the long run, insights from GWAS with cultured cell lines - ideally using peripheral blood lymphocytes or lymphoblastoid cell lines prepared from the patients themselves for whom phenotypic data on drug efficacy is available - may be beneficial for finding genetic markers for predicting the efficacy for other drug classes besides cancer drugs.

The current genome-wide technology is not perfect. Many ethnic groups have inadequate or unknown genome coverage with both Illumina (CA, USA) and Affymetrix (CA, USA) $1,000,000$ SNP chips. There is also a likely contribution of rare functional SNPs to 
important drug phenotypes. This makes personalized medicine a bit too personal, requiring extensive sequence information for each patient. However, the power offered by GWAS, if applied for combining datasets regarding drug response from several thousands of individuals, and when focused on drugs with the most severe or common adverse events, may allow the development of diagnostics offering better prediction of drug response.

\section{Bibliography}

1 Kruglyak L: The road to genome-wide association studies. Nat. Rev. Genet. 9(4), 314-318 (2008).

2 McCarthy MI, Abecasis GR, Cardon LR et al:: Genome-wide association studies for complex traits: consensus, uncertainty and challenges. Nat. Rev. Genet. 9(5), 356-369 (2008).

3 Khoury MJ, Wacholder S: Invited commentary: from genome-wide association studies to gene-environment-wide interaction studies - challenges and opportunities. Am. J. Epidemiol. 169(2), 227-230 (2009).

4 Budnitz DS, Pollock DA, Weidenbach KN, Mendelsohn AB, Schroeder TJ, Annest JL: National surveillance of emergency department visits for outpatient adverse drug events. JAMA 296(15), 1858-1866 (2006).

5 Gurwitz D, McLeod HL: Primum non nocere: adverse drug events must be taken seriously. Pharmacogenomics 8(4), 311-314 (2007).

6 Pirmohamed M, James S, Meakin S et al.: Adverse drug reactions as cause of admission to hospital: prospective analysis of 18 820 patients. BMJ 329 (7456), 15-19 (2004).

7 Need AC, Motulsky AG, Goldstein DB: Priorities and standards in pharmacogenetic research. Nat. Genet. 37(7), 671-681 (2005).

8 Giacomini KM, Krauss RM, Roden DM, Eichelbaum M, Hayden MR, Nakamura Y: When good drugs go bad. Nature 446(7139), 975-977 (2007).

\section{Financial \& competing interests disclosure}

The authors have no relevant affiliations or financial involvement with any organization or entity with a financial interest in or financial conflict with the subject matter or materials discussed in the manuscript. This includes employment, consultancies, honoraria, stock ownership or options, expert testimony, grants or patents received or pending, or royalties.

No writing assistance was utilized in the production of this manuscript.

9 Sato Y, Laird NM, Nagashima K et al.: A new statistical screening approach for finding pharmacokinetics-related genes in genome-wide studies.

Pharmacogenomics J. (2008) (Epub ahead of print).

10 Sarasquete ME, García-Sanz R, Marín L et al:: Bisphosphonate-related osteonecrosis of the jaw is associated with polymorphisms of the cytochrome P450 CYP2C8 in multiple myeloma: a genome-wide single nucleotide polymorphism analysis. Blood 112(7), 2709-2712 (2008).

11 Inada $\mathrm{T}, \mathrm{Koga} \mathrm{M}$, Ishiguro $\mathrm{H}$ et al.: Pathway-based association analysis of genome-wide screening data suggest that genes associated with the $\gamma$-aminobutyric acid receptor signaling pathway are involved in neuroleptic-induced, treatment-resistant tardive dyskinesia. Pharmacogenet. Genomics 18(4), 317-323 (2008).

12 Gurwitz D, Weizman A: Personalized psychiatry: a realistic goal. Pharmacogenomics 5(2), 213-217 (2004).

13 de Leon J: Pharmacogenomics: the promise of personalized medicine for CNS disorders. Neuropsychopharmacology 34(1), 159-172 (2009).

14 Byun E, Caillier SJ, Montalban X et al.: Genome-wide pharmacogenomic analysis of the response to interferon $\beta$ therapy in multiple sclerosis. Arch. Neurol. 65(3), 337-344 (2008).

15 Liu C, Batliwalla F, Li W et al:: Genomewide association scan identifies candidate polymorphisms associated with differential response to anti-TNF treatment in rheumatoid arthritis. Mol. Med. 14(9-10), 575-581 (2008).

16 Uhl GR, Liu QR, Drgon T et al:: Molecular genetics of successful smoking cessation: convergent genome-wide association study results. Arch. Gen. Psychiatry 65(6), 683-693 (2008).

17 Mick E, Neale B, Middleton FA, McGough JJ, Faraone SV: Genome-wide association study of response to methylphenidate in 187 children with attention-deficit/hyperactivity disorder. $A m$. J. Med. Genet. B Neuropsychiatr. Genet. 147B(8), 1412-1418 (2008).

18 Huang RS, Duan S, Kistner EO et al.: Genetic variants contributing to daunorubicin-induced cytotoxicity. Cancer Res. 68(9), 3161-3168 (2008).

19 Masri S, Phung S, Wang X et al:: Genomewide analysis of aromatase inhibitorresistant, tamoxifen-resistant, and long-term estrogen-deprived cells reveals a role for estrogen receptor. Cancer Res. 68(12), 4910-4918 (2008).

20 Greshock J, Cheng J, Rusnak D et al.: Genome-wide DNA copy number predictors of lapatinib sensitivity in tumor-derived cell lines. Mol. Cancer Ther. 7(4), 935-943 (2008). 